\title{
Clinical significance of the preoperative platelet count and platelet-to-lymphocyte ratio (PLT-PLR) in patients with surgically resected non-small cell lung cancer
}

\author{
Seok-Hyun Kim¹, Hyoun Wook Lee ${ }^{2}$, Se-Il Go ${ }^{3,4}$, Soon Il Lee ${ }^{5}$, Gyeong-Won Lee \\ ${ }^{1}$ Division of Hematology and Medical Oncology, Department of Internal Medicine, Samsung Changwon Hospital, Sungkyunkwan \\ University School of Medicine, Changwon, Republic of Korea \\ ${ }^{2}$ Department of Pathology, Samsung Changwon Hospital, Sungkyunkwan University School of Medicine, Changwon, Republic \\ of Korea \\ ${ }^{3}$ Division of Hematology-Oncology, Department of Internal Medicine, Gyeongsang National University Changwon Hospital, \\ Changwon, Republic of Korea \\ ${ }^{4}$ Division of Hematology-Oncology, Department of Internal Medicine, Gyeongsang National University Hospital, Gyeongsang \\ National University School of Medicine, Jinju, Republic of Korea \\ ${ }^{5}$ Department of Internal Medicine, Dankook University College of Medicine, Cheonan, Republic of Korea \\ ${ }^{6}$ Gyeongsang Institute of Health Sciences, Gyeongsang National University School of Medicine, Jinju, Republic of Korea \\ Correspondence to: Gyeong-Won Lee, e-mail: brightree24@gmail.com
}

Keywords: non-small cell lung cancer, platelet-to-lymphocyte ratio, thrombocytosis, inflammation, prognosis

Received: December 29, 2015

Accepted: March 31, 2016

Published: April 19, 2016

\section{ABSTRACT}

Background: The aim of this study was to assess the prognostic significance of the preoperative platelet count (PLT) and platelet-to-lymphocyte ratio (PLR) in patients with surgically resected non-small-cell lung cancer (NSCLC).

Patients and Methods: We retrospectively reviewed 202 patients treated for NSCLC between January 2002 and December 2007. Preoperative PLT and PLR scores were calculated using data obtained at the time of admission. Patients were assigned a PLT-PLR score of 0,1 , or 2 based upon the presence of thrombocytosis, an elevated PLR, or both.

Results: Patients with a PLT-PLR score of 2 had a significantly lower median overall survival (OS) [12.715 mo; 95\% confidence interval (CI) 1.215-24.215] when compared with patients with PLT-PLR scores of 1 (52.238 mo; 95\% CI 17.062$\mathbf{8 7 . 4 1 4}, p=0.002$ ) or 0 (not reached, $p<0.001$ ). Relapse-free survival (RFS) was also significantly decreased in patients with a PLT-PLR score of 2 (10.107 mo; 95\% CI 3.388-16.826) relative to patients with a PLT-PLR score of 1 (27.214 mo; 95\% CI $0-56.253, p=0.002)$ or $0(58.893 \mathrm{mo} ; 95 \%$ CI $32.938-84.848, p<0.001)$. In multivariate analysis, a PLT-PLR score of 2 was an independent prognostic factor for poor OS (hazard ratio (HR) 3.473; 95\% CI 1.765-6.835, $p<0.001$ ) and RFS (HR 2.286; 95\% CI 1.243-4.206, $p=0.008$ ) compared with a PLT-PLR score of 0.

Conclusions: Preoperative PLT-PLR scores can be useful for predicting disease prognosis in patients with surgically resected NSCLC. Further large prospective studies will be necessary to validate our findings.

\section{INTRODUCTION}

Lung cancer is the leading cause of cancer-related mortality worldwide [1]. Non-small cell lung cancer (NSCLC) accounts for approximately $85 \%$ of lung cancer cases [2]. Among NSCLC patients with resectable disease, the prognosis is dependent on the disease stage, with 5-year overall survival (OS) rates ranging from $73 \%$ for stage IA to $24 \%$ for stage IIIA disease [3]. Even in patients with pathologic stage I disease, for whom adjuvant therapy is not considered, the 5-year OS rates vary from $57 \%$ to $85 \%$ and are not satisfactory for indiscriminate exclusion of these patients from adjuvant therapy [4-6]. Therefore, a new biomarker to predict surgical outcomes is needed to 
identify patients with localized NSCLC who will benefit from adjuvant therapy.

Recent studies have demonstrated a significant role of platelets during cancer progression and metastases. Complex interactions among platelets, tumor cells, and the tumor microenvironment result in tumor growth, aberrant angiogenesis, invasion, and metastasis [7, 8]. Furthermore, reactive or paraneoplastic thrombocytosis is commonly induced by inflammation and abnormal release of cytokines in the interactive response of the host to cancer [8]. The platelet-to-lymphocyte ratio (PLR) [9-12] and thrombocytosis [13-15] have been assessed to evaluate the association between platelets and cancer progression. However, there is no consensus as to which is a more reliable marker predicting the prognosis of cancer patients.

In this study, we incorporated both markers into what we referred to as the PLT-PLR (platelet count and platelet-to-lymphocyte count ratio) score. Then, the role of the PLT-PLR score as a prognostic marker was assessed in patients with surgically resectable NSCLC.

\section{RESULTS}

\section{Patient characteristics}

This study included 202 patients with histologically confirmed, resected NSCLC. Of these, 169 (83.7\%) were male, with a median age at diagnosis of 64 years (range, 31-77 years). The most prevalent histology was squamous cell carcinoma (SqCC) $(125 / 202,61.9 \%)$. The majority of patients $(165 / 202,81.0 \%)$ had stage I or II disease, and adjuvant treatment was performed in only 57 patients $(57 / 202,28.2 \%)$. A full list of patient characteristics based on the PLT-PLR score is shown in Table 1. No statistically significant differences in terms of patient characteristics were detected between groups, with the exception of the type of surgery and T classification.

\section{Associations of the PLT, PLR, and PLT-PLR score with survival}

Both thrombocytosis and a high PLR were significantly associated with poor prognosis in the patients. Patients with thrombocytosis had a shorter OS than that of those without thrombocytosis (79.737 mo vs. $12.715 \mathrm{mo}, p<0.001)$. Patients with a high PLR also had a worse OS than that of those with a low PLR (not reached vs. $38.500 \mathrm{mo}, p=0.022$ ).

Given the significant associations of the PLT and PLR with survival of the patients, we analyzed the association between the PLT-PLR score and patient prognosis. Patients with a PLT-PLR score of 2 had a significantly poorer median OS [12.715 mo; 95\% confidence interval (CI) 1.215-24.215] when compared with patients with a PLT-PLR score of 1 (52.238 mo; 95\% CI $17.062-87.414, p=0.002$ vs. a score of 2 ) or 0 (not reached, $p<0.001$ vs. a score of 2 ; Figure 1A). Relapse- free survival (RFS) was also significantly decreased in patients with a PLT-PLR score of 2 (10.107 mo; 95\% CI 3.388-16.826) when compared with patient with a PLTPLR score of 1 (27.214 mo; 95\% CI 0-56.253, $p=0.002$ vs. a score of 2$)$ or 0 (58.893 mo; 95\% CI 32.938-84.848, $p<0.001$ vs. a score of 2; Figure 1B).

Salvage therapy after relapse did not affect the clinical outcomes of the patients in this study. In 82 patients who relapsed after surgery, there were no significant differences in OS $(p=0.777)$ or RFS $(p=0.101)$ between patients treated with any salvage therapy and those treated with BSC alone. Moreover, the proportion of patients treated with salvage therapy did not differ according to the PLT-PLR score (Table 1).

\section{Cox regression analysis}

Univariate and multivariate analyses for survival are shown in Table 2. Univariate analysis identified male sex, age $\geq 65$, SqCC, stage III disease, and a PLT-PLR score of 2 as prognostic factors for poor OS, whereas an Eastern Cooperative Oncology Group Performance Status (ECOG PS) of 1 (vs. 0), stage III disease, and a PLT-PLR score of 2 were associated with poor RFS. Multivariate analysis identified a PLT-PLR score of 2 (hazard ratio (HR) 3.473; $95 \%$ CI 1.765-6.835, $p<0.001)$ as an independent poor prognostic factor for OS. With respect to RFS, a PLT-PLR score of 2 (HR 2.286; 95\% CI 1.243-4.206, $p=0.008$ ) was also an independent poor prognostic factor in the multivariate analysis. Harrell's C-index of the Cox model that included the PLT-PLR score (OS 0.653; RFS 0.642) was higher than that in the model that did not include the PLT-PLR score (OS 0.630; RFS 0.627).

\section{Subgroup analysis}

We investigated the prognostic value of PLT-PLR relative to TNM stage, age, smoking status, sex, and histology (Table 3). A strong association between PLTPLR and OS was found irrespective of age ( $p=0.013$ for $<65 ; p<0.001$ for $\geq 65$ ), smoking status (never smoker, $p=0.007$; current or ex-smoker, $p=0.002$ ), and sex (male, $p=0.002$; female; $p=0.001$ ). The low PLT-PLR group (score 0 or 1 ) also showed a better OS relative to the high PLT-PLR group (score 2 ) for stage I/II disease ( $p=0.002$ ) and $\mathrm{SqCC}(p<0.001)$. Furthermore, a strong association between PLT-PLR and RFS was also found for stage I/ II disease $(p=0.003)$, age $\geq 65(p<0.001)$, current or ex-smoker $(p=0.002)$, male sex $(p=0.002)$, and SqCC $(p<0.001)$.

\section{DISCUSSION}

In the present study, we assessed the prognostic role of the PLT-PLR score in NSCLC patients who underwent curative surgical resection. Patients with a PLT-PLR score of 2 had very poor prognoses, with a median OS 
Table 1: Patients' characteristics according to PLT-PLR

\begin{tabular}{|c|c|c|c|c|c|c|}
\hline \multirow[b]{2}{*}{ Variables } & \multirow[b]{2}{*}{ Group } & \multirow[b]{2}{*}{$\mathbf{N}$} & \multicolumn{3}{|c|}{ PLT-PLR } & \multirow[b]{2}{*}{$\boldsymbol{P}$} \\
\hline & & & $\begin{array}{c}0 \\
(n=108, \%)\end{array}$ & $\begin{array}{c}1 \\
(n=79, \%)\end{array}$ & $\begin{array}{c}2 \\
(n=15, \%)\end{array}$ & \\
\hline Sex & $\begin{array}{l}\text { Male } \\
\text { Female }\end{array}$ & $\begin{array}{c}169 \\
33\end{array}$ & $\begin{array}{l}88(81.5) \\
20(18.5)\end{array}$ & $\begin{array}{l}67(84.8) \\
12(15.2)\end{array}$ & $\begin{array}{c}14(93.3) \\
1(6.7)\end{array}$ & 0.447 \\
\hline Age (yr) & $\begin{array}{l}<65 \\
\geq 65\end{array}$ & $\begin{array}{c}91 \\
111\end{array}$ & $\begin{array}{l}50(46.3) \\
58(53.7)\end{array}$ & $\begin{array}{l}34(43.0) \\
45(57.0)\end{array}$ & $\begin{array}{l}7(46.7) \\
8(53.3)\end{array}$ & 0.899 \\
\hline Smoking & $\begin{array}{l}\text { Never smoker } \\
\text { Current or former smoker }\end{array}$ & $\begin{array}{c}68 \\
134\end{array}$ & $\begin{array}{l}34(31.5) \\
74(68.5)\end{array}$ & $\begin{array}{l}32(40.5) \\
47(59.5)\end{array}$ & $\begin{array}{l}2(13.3) \\
13(86.7)\end{array}$ & 0.097 \\
\hline Histology & $\begin{array}{l}\mathrm{SqCC} \\
\text { Non-SqCC }\end{array}$ & $\begin{array}{c}125 \\
77\end{array}$ & $\begin{array}{l}61(56.5) \\
47(43.5)\end{array}$ & $\begin{array}{l}53(67.1) \\
26(32.9)\end{array}$ & $\begin{array}{l}11(73.3) \\
4(26.7)\end{array}$ & 0.215 \\
\hline ECOG PS & $\begin{array}{l}0 \\
1\end{array}$ & $\begin{array}{c}136 \\
66\end{array}$ & $\begin{array}{l}74(68.5) \\
34(31.5)\end{array}$ & $\begin{array}{l}52(65.8) \\
27(34.2)\end{array}$ & $\begin{array}{l}10(66.7) \\
5(33.3)\end{array}$ & 0.926 \\
\hline Surgery & $\begin{array}{l}\text { Lobectomy } \\
\text { Pneumonectomy } \\
\text { Bilobectomy or sleeve op. } \\
\text { Wedge resection } \\
\text { Segmentectomy }\end{array}$ & $\begin{array}{c}163 \\
30 \\
6 \\
2 \\
1\end{array}$ & $\begin{array}{l}95(88.0) \\
9(8.3) \\
3(2.8) \\
0(0) \\
1(0.9)\end{array}$ & $\begin{array}{c}60(75.9) \\
16(20.3) \\
2(2.5) \\
1(1.3) \\
0(0)\end{array}$ & $\begin{array}{c}8(53.3) \\
5(33.3) \\
1(6.7) \\
1(6.7) \\
0(0)\end{array}$ & $0.021 *$ \\
\hline T classification & $\begin{array}{l}\mathrm{T} 1 \mathrm{a} \text { to } \mathrm{T} 2 \mathrm{~b} \\
\mathrm{~T} 3 \text { to } \mathrm{T} 4\end{array}$ & $\begin{array}{c}178 \\
24\end{array}$ & $\begin{array}{l}98(90.7) \\
10(9.3)\end{array}$ & $\begin{array}{l}70(88.6) \\
9(11.4)\end{array}$ & $\begin{array}{l}10(66.7) \\
5(33.3)\end{array}$ & 0.026 \\
\hline $\mathbf{N}$ classification & $\begin{array}{l}\text { N0 to } \mathrm{N} 1 \\
\mathrm{~N} 2 \text { to } \mathrm{N} 3\end{array}$ & $\begin{array}{c}183 \\
19\end{array}$ & $\begin{array}{l}94(87.0) \\
14(13.0)\end{array}$ & $\begin{array}{c}75(94.9) \\
4(5.1)\end{array}$ & $\begin{array}{c}14(93.3) \\
1(6.7)\end{array}$ & 0.175 \\
\hline TNM stage & $\begin{array}{l}\text { pI } \\
\text { pII } \\
\text { pIII }\end{array}$ & $\begin{array}{l}95 \\
70 \\
37\end{array}$ & $\begin{array}{l}54(50.0) \\
35(32.4) \\
19(17.6)\end{array}$ & $\begin{array}{l}38(48.1) \\
29(36.7) \\
12(15.2)\end{array}$ & $\begin{array}{l}3(20.0) \\
6(40.0) \\
6(40.0)\end{array}$ & 0.124 \\
\hline Adjuvant therapy & $\begin{array}{l}\text { No treatment } \\
\text { Adjuvant CTx alone } \\
\text { Adjuvant CTx and RTx } \\
\text { Adjuvant RTx alone }\end{array}$ & $\begin{array}{c}145 \\
31 \\
18 \\
8\end{array}$ & $\begin{array}{c}81(75.0) \\
16(14.8) \\
8(7.4) \\
3(2.8)\end{array}$ & $\begin{array}{l}55(69.6) \\
12(15.2) \\
8(10.1) \\
4(5.1)\end{array}$ & $\begin{array}{c}9(60.0) \\
3(20.0) \\
2(13.3) \\
1(6.7)\end{array}$ & $0.883^{*}$ \\
\hline $\begin{array}{l}\text { Treatment after relapse } \\
(n=82)\end{array}$ & $\begin{array}{l}\text { CTx alone } \\
\text { RTx alone } \\
\text { CCRT } \\
\text { Re-operation } \\
\text { BSC }\end{array}$ & $\begin{array}{c}31 \\
9 \\
12 \\
2 \\
28\end{array}$ & $\begin{array}{l}18(45.0) \\
2(5.0) \\
7(17.5) \\
1(2.5) \\
12(30.0)\end{array}$ & $\begin{array}{c}10(30.3) \\
5(15.2) \\
4(12.1) \\
1(3.0) \\
13(39.4)\end{array}$ & $\begin{array}{c}3(33.3) \\
2(22.2) \\
1(11.1) \\
0(0.0) \\
3(33.3)\end{array}$ & $0.700^{\mathrm{b}}$ \\
\hline
\end{tabular}

Abbreviations: PLT, platelet; PLR, platelet-to-lymphocyte ratio; SqCC, squamous cell carcinoma; Non-SqCC, nonsquamous cell carcinoma; ECOG PS, Eastern Cooperative Oncology Group Performance Status; op, operation; CTx, chemotherapy; RTx, radiotherapy; CCRT, concurrent chemoradiotherapy; BSC, best supportive care

${ }^{a}$ Including adenocarcinoma, large cell carcinoma, bronchioalveolar carcinoma, non-small cell carcinoma.

${ }^{\mathrm{b}}$ Comparison between patients treated with any salvage therapy and with BSC alone.

*By Fisher's exact test

of only 12 months, which is similar to that reported in advanced NSCLC patients treated with palliative platinum-doublet chemotherapy [16]. The prognostic value of the PLT-PLR score was independent of age, sex, histology, and tumor stage. Furthermore, the prognosis of the patients worsened as the PLT-PLR score increased from 0 to 1 to 2. A PLT-PLR score of 1 indicates either the absence of thrombocytosis with relative lymphopenia
$\left(\mathrm{PLT} \leq 450 \times 10^{3} / \mu \mathrm{L}\right.$ and PLR $\left.>160\right)$ or the absence of relative lymphopenia with thrombocytosis (PLT $>450$ $\times 10^{3} / \mu \mathrm{L}$ and PLR $\left.\leq 160\right)$, whereas PLT-PLR scores of 0 and 2 indicate the absence and the presence of both thrombocytosis and relative lymphopenia, respectively, in most cases. These findings imply that the PLT-PLR score reflects the prognostic roles of platelets and lymphocytes more specifically compared with the PLR alone, and that 
thrombocytosis and lymphopenia may contribute equally to the poor prognosis of patients with higher PLT-PLR scores.

There is much evidence that suggests an association among platelets, lymphocytes, and tumor biology. Under normal conditions, platelets act as an important modulator of numerous physiological processes, including immune function, wound healing, and angiogenesis, as well as mediation of thrombus formation [17, 18]. However, tumor-associated angiogenesis through the release of vascular endothelial growth factor from megakaryocytes has been shown to promote tumor growth and metastasis $[7,19,20]$. Tumor cell arrest within the organ vasculature, a key process for hematogenous metastasis, is promoted by platelets [21]. Platelets promote tumor invasion by causing the breakdown of the vessel basement membrane via the release of proteolytic enzymes such as metalloproteinase-9 $[7,22]$. Furthermore, malignant tumor cells have the ability to aggregate platelets, resulting in so-called tumor cell-induced platelet aggregation (TCIPA) [23]. TCIPA allows tumor cells to evade immune surveillance and to be protected from physical clearance [23]. In contrast to platelets, lymphocytes have been associated with antitumor effects, based on the concept of 'cancer immunosurveillance'. T cells secrete cytokines and induce acute inflammation, which result in a tumor environment that enhances cytotoxic $\mathrm{T}$ cells and tissue destruction [24]. Natural killer (NK) cells also have antitumor effects through direct cytolytic activity and the production of cytokines $[25,26]$. In various types of cancer, increased infiltration of tumor-infiltrating lymphocytes (TILs) is associated with a good prognosis and favorable responses

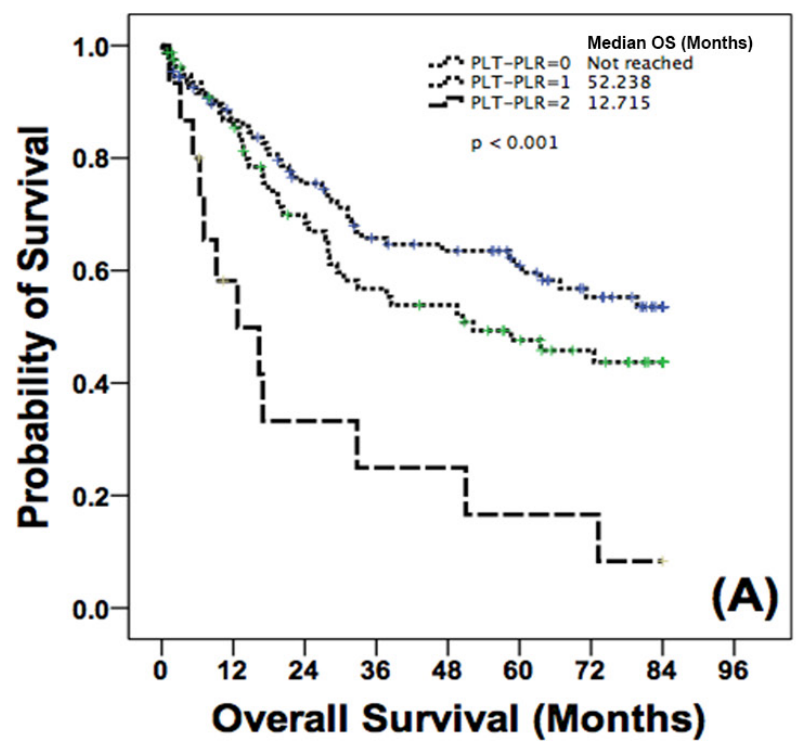

to anticancer therapy [27-29]. This evidence, which is in opposition to the effects of platelets and lymphocytes on tumor biology, supports our suggestion that a marker incorporating both values is needed for a more reliable prediction of prognosis in cancer patients.

Several lines of evidence suggest a relationship between PLR and survival across several types of cancer. A recent Chinese study examining pretreatment PLR scores in 210 advanced NSCLC patients suggested the PLR may be useful to predict disease outcomes and the response to first-line chemotherapy [10]. In this study, an elevated PLR was associated with a 2-fold risk of death and a 4.5 -fold risk of early progression. Similar findings were observed in a previous study that evaluated 372 stage II-III colon cancer patients who underwent surgery [30]. In that study, the patients with an elevated PLR had a $65 \%$ increased risk of recurrence. A large Austrian study examining 793 non-metastatic breast cancer patients showed that an elevated preoperative PLR increases the risk of death two-fold [11]. Taken together, these data suggest that an elevated PLR is significantly related to poor prognosis in patients with cancer. In addition to the clinical significance of PLR, its high accessibility makes this marker useful in clinical practice. Complete blood counts are obtained in all patients undergoing planned surgery, meaning that the PLR can be measured easily in nearly all patients, thereby eliminating the need for additional tests to obtain this marker. Moreover, this approach is also faster and cheaper than other conventional markers such as serum CEA, CA 19-9, SCC, NSE, and CYFRA 21-1. However, measuring the PLR alone has a disadvantage in some cases. For example, in the presence

Figure 1: Kaplan-Meier curves for A. OS and B. RFS according to PLT-PLR. 
Table 2: Cox proportional regression model for OS and RFS

\begin{tabular}{|c|c|c|c|c|c|c|c|c|c|c|c|c|}
\hline \multirow{3}{*}{ Variables } & \multicolumn{6}{|c|}{ OS } & \multicolumn{6}{|c|}{ RFS } \\
\hline & \multicolumn{3}{|c|}{ Univariate } & \multicolumn{3}{|c|}{ Multivariate } & \multicolumn{3}{|c|}{ Univariate } & \multicolumn{3}{|c|}{ Multivariate } \\
\hline & HR & $95 \%$ CI & $P$ & HR & $95 \% \mathrm{CI}$ & $P$ & HR & $95 \%$ CI & $P$ & HR & $95 \%$ CI & $P$ \\
\hline $\begin{array}{l}\text { Sex } \\
\quad \text { Male } \\
\text { Female }\end{array}$ & $\begin{array}{c}\text { Reference } \\
0.477\end{array}$ & $\begin{array}{c}0.247- \\
0.919\end{array}$ & 0.027 & $\begin{array}{c}\text { Reference } \\
0.655\end{array}$ & $\begin{array}{c}0.331- \\
1.296\end{array}$ & 0.224 & $\begin{array}{c}\text { Reference } \\
0.727\end{array}$ & $\begin{array}{c}0.435- \\
1.216\end{array}$ & 0.224 & & & \\
\hline $\begin{array}{l}\text { Age (yr) } \\
\quad<65 \\
\quad \geq 65\end{array}$ & $\begin{array}{c}\text { Reference } \\
1.666\end{array}$ & $\begin{array}{l}1.090- \\
2.545\end{array}$ & 0.018 & $\begin{array}{c}\text { Reference } \\
2.038\end{array}$ & $\begin{array}{l}1.322- \\
3.143\end{array}$ & 0.001 & $\begin{array}{c}\text { Reference } \\
1.316\end{array}$ & $\begin{array}{c}0.909- \\
1.905\end{array}$ & 0.146 & & & \\
\hline $\begin{array}{l}\text { Smoking } \\
\text { Never smoker } \\
\text { Current or } \\
\text { former smoker }\end{array}$ & $\begin{array}{c}\text { Reference } \\
0.835\end{array}$ & $\begin{array}{c}0.624- \\
1.465\end{array}$ & 0.956 & & & & $\begin{array}{c}\text { Reference } \\
0.962\end{array}$ & $\begin{array}{c}0.657- \\
1.409\end{array}$ & 0.842 & & & \\
\hline $\begin{array}{l}\text { Histology } \\
\text { SqCC } \\
\text { Non-SqCC }\end{array}$ & $\begin{array}{c}\text { Reference } \\
0.623\end{array}$ & $\begin{array}{c}0.400- \\
0.969\end{array}$ & 0.036 & $\begin{array}{c}\text { Reference } \\
0.911\end{array}$ & $\begin{array}{c}0.571- \\
1.456\end{array}$ & 0.698 & $\begin{array}{c}\text { Reference } \\
0.741\end{array}$ & $\begin{array}{c}0.505- \\
1.087\end{array}$ & 0.126 & & & \\
\hline $\begin{array}{c}\text { ECOG PS } \\
0 \\
1\end{array}$ & $\begin{array}{c}\text { Reference } \\
1.414\end{array}$ & $\begin{array}{c}0.932- \\
2.146\end{array}$ & 0.104 & & & & $\begin{array}{c}\text { Reference } \\
1.509\end{array}$ & $\begin{array}{l}1.041- \\
2.187\end{array}$ & 0.030 & $\begin{array}{c}\text { Reference } \\
1.443\end{array}$ & $\begin{array}{c}0.985- \\
2.113\end{array}$ & 0.060 \\
\hline $\begin{array}{l}\text { Surgery } \\
\text { Lobectomy and } \\
\text { others } \\
\text { Pneumonectomy }\end{array}$ & $\begin{array}{c}\text { Reference } \\
1.147\end{array}$ & $\begin{array}{c}0.660- \\
1.993\end{array}$ & 0.626 & & & & $\begin{array}{c}\text { Reference } \\
1.361\end{array}$ & $\begin{array}{c}0.849- \\
2.182\end{array}$ & 0.201 & & & \\
\hline $\begin{array}{l}\text { TNM stage } \\
\text { pI } \\
\text { pII } \\
\text { pIII }\end{array}$ & $\begin{array}{c}\text { Reference } \\
1.439 \\
2.260\end{array}$ & $\begin{array}{l}0.922- \\
2.246 \\
1.318- \\
3.876\end{array}$ & $\begin{array}{l}0.109 \\
0.003\end{array}$ & $\begin{array}{c}\text { Reference } \\
1.400 \\
2.222\end{array}$ & $\begin{array}{l}0.887- \\
2.210 \\
1.248- \\
3.954\end{array}$ & $\begin{array}{l}0.149 \\
0.007\end{array}$ & $\begin{array}{c}\text { Reference } \\
1.636 \\
2.950\end{array}$ & $\begin{array}{l}1.096- \\
2.442 \\
1.812- \\
4.804\end{array}$ & $\begin{array}{c}0.016 \\
<0.001\end{array}$ & $\begin{array}{c}\text { Reference } \\
1.628 \\
2.350\end{array}$ & $\begin{array}{l}1.086- \\
2.438 \\
1.394- \\
3.962\end{array}$ & $\begin{array}{l}0.018 \\
0.001\end{array}$ \\
\hline $\begin{array}{l}\text { PLT-PLR } \\
0 \\
1 \\
2\end{array}$ & $\begin{array}{c}\text { Reference } \\
1.360 \\
3.787\end{array}$ & $\begin{array}{l}0.882- \\
2.099 \\
1.986- \\
7.220\end{array}$ & $\begin{array}{c}0.148 \\
<0.001\end{array}$ & $\begin{array}{c}\text { Reference } \\
1.356 \\
3.473\end{array}$ & $\begin{array}{l}0.886- \\
2.075 \\
1.765- \\
6.835\end{array}$ & $\begin{array}{c}0.161 \\
< \\
0.001\end{array}$ & $\begin{array}{c}\text { Reference } \\
1.204 \\
3.087\end{array}$ & $\begin{array}{c}0.818- \\
1.774 \\
1.712- \\
5.565\end{array}$ & $\begin{array}{c}0.310 \\
<0.001\end{array}$ & $\begin{array}{c}\text { Reference } \\
1.248 \\
2.286\end{array}$ & $\begin{array}{c}0.853- \\
1.828 \\
1.243- \\
4.206\end{array}$ & $\begin{array}{l}0.254 \\
0.008\end{array}$ \\
\hline
\end{tabular}

Abbreviations: OS, overall survival; RFS, relapse-free survival; HR, hazard ratio; 95\% CI, 95\% confidence interval; SqCC, squamous cell carcinoma; Non-SqCC, non-squamous cell carcinoma; ECOG PS, Eastern Cooperative Oncology Group Performance Status; PLT, platelet; PLR, platelet-to-lymphocyte ratio

Table 3: Subgroup analysis for OS and RFS according to PLT-PLR score

\begin{tabular}{|c|c|c|c|c|c|c|c|c|}
\hline & \multirow{2}{*}{ PLT-PLR } & \multirow{2}{*}{ N (\%) } & \multicolumn{3}{|c|}{ OS, months } & \multicolumn{3}{|c|}{ RFS, months } \\
\hline & & & Median (SD) & $95 \% \mathrm{CI}$ & $P$ & Median (SD) & $95 \% \mathrm{CI}$ & $P$ \\
\hline \multicolumn{9}{|l|}{ Stage } \\
\hline \multirow{4}{*}{$\mathrm{pI}$ and $\mathrm{pII}$} & 0 & $89(53.9)$ & $61.014(3.377)$ & $\begin{array}{c}54.395- \\
67.632\end{array}$ & 0.002 & $53.384(3.619)$ & $\begin{array}{c}46.291- \\
60.477\end{array}$ & 0.003 \\
\hline & 1 & $67(40.6)$ & $51.197(4.062)$ & $\begin{array}{l}43.236- \\
59.158\end{array}$ & & $43.905(4.290)$ & $\begin{array}{c}35.497- \\
52.313\end{array}$ & \\
\hline & 2 & $9(5.5)$ & $27.695(9.395)$ & $\begin{array}{l}9.282- \\
46.108\end{array}$ & & $18.370(8.079)$ & $\begin{array}{l}2.535- \\
34.205\end{array}$ & \\
\hline & 0 & $19(51.4)$ & $37.717(18.733)$ & $\begin{array}{l}1.001- \\
74.433\end{array}$ & 0.221 & $11.663(4.451)$ & $\begin{array}{l}2.939- \\
20.388\end{array}$ & 0.724 \\
\hline \multirow[t]{2}{*}{ pIII } & 1 & $12(32.4)$ & $38.500(17.175)$ & $\begin{array}{l}4.837- \\
72.163\end{array}$ & & $12.057(5.620)$ & $\begin{array}{l}1.042- \\
23.073\end{array}$ & \\
\hline & 2 & $6(16.2)$ & $9.166(3.106)$ & $\begin{array}{l}3.078- \\
15.255\end{array}$ & & $6.505(2.286)$ & $\begin{array}{l}2.024- \\
10.986\end{array}$ & \\
\hline
\end{tabular}

(Continued) 


\begin{tabular}{|c|c|c|c|c|c|c|c|c|}
\hline & \multirow{2}{*}{ PLT-PLR } & \multirow{2}{*}{ N (\%) } & \multicolumn{3}{|c|}{ OS, months } & \multicolumn{3}{|c|}{ RFS, months } \\
\hline & & & Median (SD) & $95 \% \mathrm{CI}$ & $P$ & Median (SD) & $95 \% \mathrm{CI}$ & $\boldsymbol{P}$ \\
\hline \multicolumn{9}{|l|}{ Age (yr) } \\
\hline \multirow{4}{*}{$<65$} & 0 & $50(54.9)$ & $64.991(4.407)$ & $\begin{array}{l}56.353- \\
73.628\end{array}$ & 0.013 & $53.127(5.051)$ & $\begin{array}{l}43.227- \\
63.028\end{array}$ & 0.207 \\
\hline & 1 & $34(37.4)$ & $55.156(6.092)$ & $\begin{array}{c}43.216- \\
67.096\end{array}$ & & $44.467(6.610)$ & $\begin{array}{c}31.512- \\
57.422\end{array}$ & \\
\hline & 2 & $7(7.7)$ & $37.428(11.515)$ & $\begin{array}{c}14.858- \\
59.998\end{array}$ & & $30.329(11.378)$ & $\begin{array}{l}8.027- \\
52.630\end{array}$ & \\
\hline & 0 & $58(52.3)$ & $66.793(23.153)$ & $\begin{array}{l}21.412- \\
112.173\end{array}$ & $\begin{array}{c}< \\
0.001\end{array}$ & $40.214(18.758)$ & $\begin{array}{l}3.449- \\
76.980\end{array}$ & $<0.001$ \\
\hline \multirow[t]{2}{*}{$\geq 65$} & 1 & $45(40.5)$ & $50.464(17.928)$ & $\begin{array}{l}15.325- \\
85.604\end{array}$ & & $27.214(18.203)$ & $\begin{array}{l}0.000- \\
62.891\end{array}$ & \\
\hline & 2 & $8(7.2)$ & $9.166(3.505)$ & $\begin{array}{l}2.297- \\
16.036\end{array}$ & & $6.374(1.165)$ & $\begin{array}{l}4.090- \\
8.658\end{array}$ & \\
\hline \multicolumn{9}{|l|}{ Smoking } \\
\hline & 0 & $34(50.0)$ & $58.563(5.396)$ & $\begin{array}{l}47.988- \\
69.138\end{array}$ & 0.007 & $47.126(5.770)$ & $\begin{array}{l}35.816- \\
58.436\end{array}$ & 0.108 \\
\hline \multirow[t]{3}{*}{$\begin{array}{l}\text { Never } \\
\text { smoker }\end{array}$} & 1 & $32(47.1)$ & $51.704(6.285)$ & $\begin{array}{c}39.386- \\
64.022\end{array}$ & & $45.813(6.529)$ & $\begin{array}{l}33.016- \\
58.609\end{array}$ & \\
\hline & 2 & $2(2.9)$ & $9.544(3.170)$ & $\begin{array}{l}3.330- \\
15.758\end{array}$ & & 9.544(3.170) & $\begin{array}{l}3.330- \\
15.758\end{array}$ & \\
\hline & 0 & $74(55.2)$ & $58.121(3.960)$ & $\begin{array}{l}50.359- \\
65.884\end{array}$ & 0.002 & $49.736(4.190)$ & $\begin{array}{l}41.524- \\
57.948\end{array}$ & 0.002 \\
\hline \multirow[t]{2}{*}{$\begin{array}{l}\text { Current or } \\
\text { Ex-smoker }\end{array}$} & 1 & $47(35.1)$ & $50.277(4.826)$ & $\begin{array}{l}40.818- \\
59.736\end{array}$ & & $40.399(5.081)$ & $\begin{array}{c}30.441- \\
50.356\end{array}$ & \\
\hline & 2 & $13(9.7)$ & $29.472(8.539)$ & $\begin{array}{l}12.737- \\
46.208\end{array}$ & & $19.524(6.987)$ & $\begin{array}{l}5.830- \\
33.218\end{array}$ & \\
\hline \multicolumn{9}{|l|}{ Sex } \\
\hline \multirow{4}{*}{ Male } & 0 & $88(52.1)$ & $55.027(3.673)$ & $\begin{array}{l}47.829- \\
62.226\end{array}$ & 0.002 & $47.102(3.833)$ & $\begin{array}{l}39.589- \\
54.615\end{array}$ & 0.002 \\
\hline & 1 & $67(39.6)$ & 49.096(4.189) & $\begin{array}{l}40.885- \\
57.307\end{array}$ & & $41.603(4.378)$ & $\begin{array}{c}33.023- \\
50.184\end{array}$ & \\
\hline & 2 & $14(8.3)$ & $27.212(8.136)$ & $\begin{array}{l}11.265- \\
43.159\end{array}$ & & $18.658(6.545)$ & $\begin{array}{l}5.829- \\
31.486\end{array}$ & \\
\hline & 0 & $20(60.6)$ & $71.272(5.302)$ & $\begin{array}{l}60.880- \\
81.664\end{array}$ & 0.001 & $65.676(2.783)$ & $\begin{array}{l}60.220- \\
71.131\end{array}$ & 0.099 \\
\hline \multirow[t]{2}{*}{ Female } & 1 & $12(36.4)$ & $61.945(8.848)$ & $\begin{array}{l}44.603- \\
79.286\end{array}$ & & 33.857 & & \\
\hline & 2 & $1(3.0)$ & 16.953 & & & 11.696 & & \\
\hline
\end{tabular}

(Continued) 


\begin{tabular}{|c|c|c|c|c|c|c|c|c|}
\hline & \multirow{2}{*}{ PLT-PLR } & \multirow{2}{*}{ N (\%) } & \multicolumn{3}{|c|}{ OS, months } & \multicolumn{3}{|c|}{ RFS, months } \\
\hline & & & Median (SD) & $95 \% \mathrm{CI}$ & $P$ & Median (SD) & $95 \% \mathrm{CI}$ & $P$ \\
\hline \multicolumn{9}{|l|}{ Histology } \\
\hline \multirow{3}{*}{$\mathrm{SqCC}$} & 0 & $61(48.8)$ & $57.701(4.282)$ & $\begin{array}{l}49.308- \\
66.095\end{array}$ & $\begin{array}{c}< \\
0.001\end{array}$ & $50.523(4.510)$ & $\begin{array}{l}41.684- \\
59.362\end{array}$ & $<0.001$ \\
\hline & 1 & $53(42.4)$ & $43.592(4.575)$ & $\begin{array}{l}34.626- \\
52.559\end{array}$ & & $34.520(4.601)$ & $\begin{array}{l}25.501- \\
43.538\end{array}$ & \\
\hline & 2 & $11(8.8)$ & $20.987(7.558)$ & $\begin{array}{l}6.172- \\
35.802\end{array}$ & & $14.913(5.573)$ & $\begin{array}{l}3.990- \\
25.836\end{array}$ & \\
\hline \multirow{3}{*}{ Non-SqCC } & 0 & $47(61.0)$ & $58.616(4.824)$ & $\begin{array}{c}49.161- \\
68.071\end{array}$ & 0.096 & $46.457(5.126)$ & $\begin{array}{l}36.411- \\
56.503\end{array}$ & 0.098 \\
\hline & 1 & $26(33.8)$ & $66.095(5.900)$ & $\begin{array}{l}54.531- \\
77.659\end{array}$ & & $59.314(6.731)$ & $\begin{array}{c}46.121- \\
72.507\end{array}$ & \\
\hline & 2 & $4(5.2)$ & $35.368(17.107)$ & $\begin{array}{l}1.839- \\
68.897\end{array}$ & & $27.215(16.922)$ & $\begin{array}{l}0.000- \\
60.382\end{array}$ & \\
\hline
\end{tabular}

Abbreviations: PLT, platelet; PLR, platelet-to-lymphocyte ratio; SqCC, squamous cell carcinoma; Non-SqCC, nonsquamous cell carcinoma

of severe lymphopenia, the PLR may increase even in patients without thrombocytosis. In the presence of lymphocytosis, the PLR may decrease even in patients with thrombocytosis. Instead, as described above, the PLT-PLR score can discriminate these ambiguous cases by appointing a score of 1 , which suggests an intermediate prognosis.

As with all studies, this work has several limitations, which should be taken into consideration. First, the sample size was relatively small for generalizing the clinical significance of the PLT-PLR in resectable NSCLC. Moreover, among the 202 patients enrolled, the number of patients with a PLT-PLR score of 2 , which is associated with the worst prognosis, was only $15(7.4 \%)$. Therefore, our data must be regarded as preliminary. In particular, the subgroup analysis (Table 3) should be interpreted with caution. Confirmation of the present results in an independent data set is imperative for drawing firm conclusions about the role of PLT-PLR for NSCLC prognosis. Second, the cut-off values used for each marker comprising the PLT-PLR score were not confirmative. The cut-off value for the PLR was determined using a minimal $p$-value approach, which leads to inflation of the type I error rate [31]. The PLT cut-off value of $450 \times$ $10^{3} / \mu \mathrm{L}$ is commonly used in clinical practice. Although the prognosis of the patients was significantly influenced by either the PLT or PLR alone, further analyses are necessary to establish cut-off values for each marker.

In conclusion, based on the PLT-PLR scoring system, patients with resectable NSCLC could be classified into three groups with different prognoses in this study. This platelet- and lymphocyte-based prognostic model may be useful for predicting postoperative outcomes and for individualizing postoperative management plans in patients with surgically resectable NSCLC. A large prospective study is needed to properly validate our findings.

\section{MATERIALS AND METHODS}

\section{Study population}

We retrospectively reviewed all patients histologically confirmed for NSCLC who were treated by surgical resection at Gyeongsang National University Hospital Regional Cancer Center (Jinju, Korea) and Samsung Changwon Hospital (Changwon, Korea) between January 2002 and December 2007. Inclusion criteria were age $\geq 18$ years, an ECOG PS of 0 or 1, and adequate hematologic, liver, and kidney function. Patients were excluded if they exhibited clinical evidence of infection or other inflammatory conditions, or were treated previously with neoadjuvant chemotherapy or radiotherapy. Our Institutional Review Board approved this retrospective study (2012-SCMC-034-00) and waived the requirement for informed consent. Baseline characteristics including demographics, smoking status, performance status, and medical history were collected using an electronic medical record system. Complete blood cell counts with differential counts at diagnosis were evaluated.

\section{Diagnostic criteria}

The PLR was defined as the absolute PLT divided by the absolute lymphocyte count. The optimal cut-off value for PLR was determined using a minimal $p$-value 
approach. PLR values were categorized into two groups: $<160$ and $\geq 160$. A PLT $>450 \times 10^{3} / \mu \mathrm{L}$ was defined as thrombocytosis. Patients were assigned a PLT-PLR score of 0,1 , or 2 based on the presence of thrombocytosis, an elevated PLR ( $>160)$, or both, as follows: patients with both thrombocytosis and an elevated PLR were assigned a score of 2, and patients with either or neither were assigned a score of 1 or 0 , respectively.

\section{Statistical analysis}

The association between clinicopathological parameters and the PLT-PLR was analyzed using the $\chi^{2}$ or Fisher's exact test, as appropriate. OS was calculated from the date of diagnosis to the date of death from any cause or the date of the last follow-up observation. RFS was calculated from the date of diagnosis to the date of recurrence or the date of death from any cause. Patients who did not die during the course of follow-up were censored at the date last seen alive. Kaplan-Meier analysis and log rank tests were used to compare the postoperative survival curves between groups. Univariate and multivariate analyses of survival were conducted using the Cox proportional hazards model with the Enter selection method. All variables with $p<0.1$ in the univariate analyses were included in the multivariate analysis. The predictive ability of the models was evaluated using Harrell's C-index. $P$ values $<0.05$ were considered statistically significant. All statistical analyses were conducted using SPSS for Windows (ver. 18.0; SPSS Inc., Chicago, IL, USA) and STATA software (ver. 14.0; StataCorp, College Station, TX, USA).

\section{ACKNOWLEDGMENTS AND FUNDING}

No funding is associated with this work.

\section{CONFLICTS OF INTEREST}

The authors declare no conflicts of interest.

\section{REFERENCES}

1. Siegel RL, Miller KD, Jemal A. Cancer statistics, 2015. CA Cancer J Clin. 2015; 65: 5-29.

2. Hainsworth JD, Cebotaru CL, Kanarev V, Ciuleanu TE, Damyanov D, Stella P, Ganchev H, Pover G, Morris C, Tzekova V. A phase II, open-label, randomized study to assess the efficacy and safety of AZD6244 (ARRY-142886) versus pemetrexed in patients with non-small cell lung cancer who have failed one or two prior chemotherapeutic regimens. J Thorac Oncol. 2010; 5: 1630-1636.

3. Goldstraw P, Crowley J, Chansky K, Giroux DJ, Groome PA, Rami-Porta R, Postmus PE, Rusch V, Sobin L, International Association for the Study of Lung Cancer International Staging C, Participating I. The IASLC Lung
Cancer Staging Project: proposals for the revision of the TNM stage groupings in the forthcoming (seventh) edition of the TNM Classification of malignant tumours. J Thorac Oncol. 2007; 2: 706-714.

4. Bott MJ, Patel AP, Crabtree TD, Colditz GA, Kreisel D, Krupnick AS, Patterson GA, Broderick S, Meyers BF, Puri V. Pathologic Upstaging in Patients Undergoing Resection for Stage I Non-Small Cell Lung Cancer: Are There Modifiable Predictors? Ann Thorac Surg. 2015; 100: 2048-2053.

5. Okami J, Ito Y, Higashiyama M, Nakayama T, Tokunaga T, Maeda J, Kodama K. Sublobar resection provides an equivalent survival after lobectomy in elderly patients with early lung cancer. Ann Thorac Surg. 2010; 90: 1651-1656.

6. Scheel PJ, 3rd, Crabtree TD, Bell JM, Frederiksen C, Broderick SR, Krupnick AS, Kreisel D, Patterson GA, Meyers BF, Puri V. Does surgeon experience affect outcomes in pathologic stage I lung cancer? J Thorac Cardiovasc Surg. 2015; 149: 998-1004 e1001.

7. Bambace NM, Holmes CE. The platelet contribution to cancer progression. J Thromb Haemost. 2011; 9: 237-249.

8. Goubran HA, Stakiw J, Radosevic M, Burnouf T. Plateletcancer interactions. Semin Thromb Hemost. 2014; 40: 296-305.

9. Kos M, Hocazade C, Kos FT, Uncu D, Karakas E, Dogan M, Uncu HG, Yildirim N, Zengin N. Prognostic role of pretreatment platelet/lymphocyte ratio in patients with nonsmall cell lung cancer. Wien Klin Wochenschr. 2015 Feb 27. PMID: 25720573

10. Liu H, Wu Y, Wang Z, Yao Y, Chen F, Zhang H, Wang Y, Song Y. Pretreatment platelet-to-lymphocyte ratio (PLR) as a predictor of response to first-line platinum-based chemotherapy and prognosis for patients with non-small cell lung cancer. J Thorac Dis. 2013; 5: 783-789.

11. Krenn-Pilko S, Langsenlehner U, Thurner EM, Stojakovic T, Pichler M, Gerger A, Kapp KS, Langsenlehner T. The elevated preoperative platelet-to-lymphocyte ratio predicts poor prognosis in breast cancer patients. Br J Cancer. 2014; 110: 2524-2530.

12. Smith RA, Bosonnet L, Raraty M, Sutton R, Neoptolemos JP, Campbell F, Ghaneh P. Preoperative plateletlymphocyte ratio is an independent significant prognostic marker in resected pancreatic ductal adenocarcinoma. Am J Surg. 2009; 197: 466-472.

13. Kim M, Chang H, Yang HC, Kim YJ, Lee CT, Lee JH, Jheon S, Kim K, Chung JH, Lee JS. Preoperative thrombocytosis is a significant unfavorable prognostic factor for patients with resectable non-small cell lung cancer. World J Surg Oncol. 2014; 12: 37.

14. Tomita M, Shimizu T, Hara M, Ayabe T, Onitsuka T. Prognostic impact of thrombocytosis in resectable nonsmall cell lung cancer. Interact Cardiovasc Thorac Surg. 2008; 7: 613-615.

15. Kim KH, Park TY, Lee JY, Lee SM, Yim JJ, Yoo CG, Kim YW, Han SK, Yang SC. Prognostic significance of initial 
platelet counts and fibrinogen level in advanced non-small cell lung cancer. J Korean Med Sci. 2014; 29: 507-511.

16. Scagliotti GV, Parikh P, von Pawel J, Biesma B, Vansteenkiste J, Manegold C, Serwatowski P, Gatzemeier U, Digumarti R, Zukin M, Lee JS, Mellemgaard A, Park K et al. Phase III study comparing cisplatin plus gemcitabine with cisplatin plus pemetrexed in chemotherapy-naive patients with advanced-stage non-small-cell lung cancer. J Clin Oncol. 2008; 26: 3543-3551.

17. Morrell CN, Aggrey AA, Chapman LM, Modjeski KL. Emerging roles for platelets as immune and inflammatory cells. Blood. 2014; 123: 2759-2767.

18. Walsh TG, Metharom P, Berndt MC. The functional role of platelets in the regulation of angiogenesis. Platelets. 2015; 26: 199-211.

19. Dvorak HF, Brown LF, Detmar M, Dvorak AM. Vascular permeability factor/vascular endothelial growth factor, microvascular hyperpermeability, and angiogenesis. Am J Pathol. 1995; 146: 1029-1039.

20. Ma L, Perini R, McKnight W, Dicay M, Klein A, Hollenberg MD, Wallace JL. Proteinase-activated receptors 1 and 4 counter-regulate endostatin and VEGF release from human platelets. Proc Natl Acad Sci U S A. 2005; 102: 216-220.

21. Lewalle JM, Castronovo V, Goffinet G, Foidart JM. Malignant cell attachment to endothelium of ex vivo perfused human umbilical vein. Modulation by platelets, plasma and fibronectin. Thromb Res. 1991; 62: 287-298.

22. Huang S, Van Arsdall M, Tedjarati S, McCarty M, Wu W, Langley R, Fidler IJ. Contributions of stromal metalloproteinase-9 to angiogenesis and growth of human ovarian carcinoma in mice. J Natl Cancer Inst. 2002; 94: 1134-1142.

23. Jurasz P, Alonso-Escolano D, Radomski MW. Plateletcancer interactions: mechanisms and pharmacology of tumour cell-induced platelet aggregation. Br J Pharmacol. 2004; 143: 819-826.
24. Disis ML. Immune regulation of cancer. J Clin Oncol. 2010; 28: 4531-4538.

25. Menard C, Blay JY, Borg C, Michiels S, Ghiringhelli F, Robert C, Nonn C, Chaput N, Taieb J, Delahaye NF, Flament C, Emile JF, Le Cesne A et al. Natural killer cell IFN-gamma levels predict long-term survival with imatinib mesylate therapy in gastrointestinal stromal tumor-bearing patients. Cancer Res. 2009; 69: 3563-3569.

26. Vivier E, Ugolini S, Blaise D, Chabannon C, Brossay L. Targeting natural killer cells and natural killer T cells in cancer. Nat Rev Immunol. 2012; 12: 239-252.

27. Loi S, Sirtaine N, Piette F, Salgado R, Viale G, Van Eenoo F, Rouas G, Francis P, Crown JP, Hitre E, de Azambuja E, Quinaux E, Di Leo A et al. Prognostic and predictive value of tumor-infiltrating lymphocytes in a phase III randomized adjuvant breast cancer trial in node-positive breast cancer comparing the addition of docetaxel to doxorubicin with doxorubicin-based chemotherapy: BIG 02-98. J Clin Oncol. 2013; 31: 860-867.

28. Balermpas P, Rodel F, Weiss C, Rodel C, Fokas E. Tumor-infiltrating lymphocytes favor the response to chemoradiotherapy of head and neck cancer. Oncoimmunology. 2014; 3: e27403.

29. Mahmoud SM, Paish EC, Powe DG, Macmillan RD, Grainge MJ, Lee AH, Ellis IO, Green AR. Tumorinfiltrating CD8+ lymphocytes predict clinical outcome in breast cancer. J Clin Oncol. 2011; 29: 1949-1955.

30. Szkandera J, Pichler M, Absenger G, Stotz M, Arminger F, Weissmueller M, Schaberl-Moser R, Samonigg H, Kornprat P, Stojakovic T, Avian A, Gerger A. The elevated preoperative platelet to lymphocyte ratio predicts decreased time to recurrence in colon cancer patients. Am J Surg. 2014; 208: 210-214.

31. Altman DG, Lausen B, Sauerbrei W, Schumacher M. Dangers of using "optimal" cutpoints in the evaluation of prognostic factors. J Natl Cancer Inst. 1994; 86: 829-835. 